\title{
Optimization Design of Magnetic Gear Based on Genetic Algorithm Toolbox of Matlab
}

\author{
Li-Bing Jing ${ }^{\dagger}$, Zheng-Hao Luo*, Lin Liu* and Qi-Xing Gao*
}

\begin{abstract}
Concentric magnetic gear (CMG) is a transmission gear with a novel structure and a promising prospect. However, the calculation of its electromagnetic field is more complicated. In this paper, an exact analytical method is used to calculate the magnetic field distribution and electromagnetic torque. The genetic algorithm optimization toolbox of matlab (GAOT) provides a good tool for its optimization design. The optimization results show that the genetic algorithm is practicable and effective, and provide a new approach for the analysis and design of the concentric magnetic gear. It is significant for the research and application of the concentric magnetic gear.
\end{abstract}

Keywords: Concentric magnetic gear, Exact analytical method, Genetic algorithm, Optimization design

\section{Introduction}

Concentric magnetic gear is a drive device that transmits the magnetic force through non-mechanical contact with characteristics of low energy consumption, high torque density, high reliability, long service life. It has a promising prospect in the low-speed and high-torque respects of ships(including submarine) [1], electric locomotives [2], electric vehicle [3], elevators [4], wind power generation [5], tidal power generation [6].

An accurate knowledge of the air-gap magnetic field distribution is necessary for predicting the performance of the magnetic gear. The air-gap magnetic field can be evaluated by analytical or semi-analytical methods or by numerical techniques like finite elements. Finite elements give accurate results considering geometric details and nonlinearity of magnetic materials. However, this method is computer time consuming and poorly flexible for the first step of design stage of a magnetic gear. Analytical methods are useful tools for first evaluation of magnetic gear performances and for design optimization since continuous derivatives issued from the analytical solution are of great importance in most optimization methods.

As shown in Fig. 1, the structure model of the concentric magnetic gear has two layers of air gap fields whose calculation is complicated. Because of open slots on two sides, so it is difficult to handle in the analytical prediction of the air-gap magnetic field. Analytical approaches for airgap magnetic field computation in slotted machines can be found in the literature [7-10]. However, the publications

$\dagger$ Corresponding Author: Hubei Key Laboratory of Hydroelectric Machinery Design \& Maintenance, China Three Gorges University, Yichang, China. (jinglibing163@163.com)

* College of Electrical Engineering \& New Energy, China Three Gorges University, Yichang, China. (\{lzh910823, liulinmiyang\} @163.com, gaoqixing2015@sina.com)

Received: November 1, 2014; Accepted: March 21, 2016

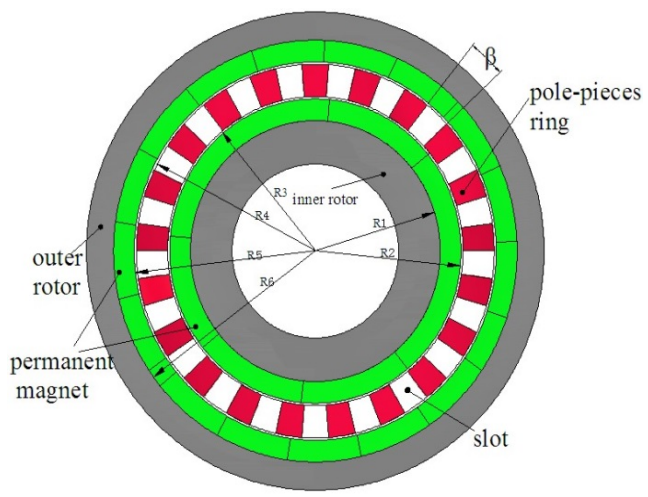

Fig. 1. Geometry of the concentric magnetic gear

focus essentially on the determination of cogging torque in permanent magnet motors.

In order to improve motor performance, many researchers are conducting tests for the optimization of engine parameters with Genetic Algorithm. In [11], the authors optimized a permanent magnet brushless dc motor with Genetic Algorithm, and considered the slot electric loading, magnet-fraction, slot-fraction, air-gap, and air-gap flux density as the design variables. In [12], the Genetic Algorithm is used to optimize the distribution of the PMs and minimize the cogging torque of the motor. In [13], the authors reported an axial-flux permanent magnet motor optimized by the Genetic Algorithm-based sizing equation and the finite-element analysis, FEA and GA simulation results were compared and agreed well to the flux density in various areas of the designed motor at no-load condition.

To the authors' knowledge, no literature on the analytical solution for optimization design of magnetic gear based on Genetic Algorithm has been published yet. In this paper, the exact analytical method is adopted to analyze and calculate the electromagnetic fields and the Matlab genetic algorithm is employed to optimize the parameters. 
Table 1. The meaning of parameters

\begin{tabular}{c|l}
\hline Symbol & \multicolumn{1}{c}{ Quantity } \\
\hline$R_{1}$ & Radius of the inner rotor yoke \\
\hline$R_{2}$ & Outer radius of the inner rotor PMs \\
\hline$R_{3}$ & Inner radius of the slots \\
\hline$R_{4}$ & Outer radius of the slots \\
\hline$R_{5}$ & Inner radius of the outer rotor PMs \\
\hline$R_{6}$ & Inner radius of the outer rotor yoke \\
\hline$\beta$ & Slot opening \\
\hline
\end{tabular}

The geometrical parameters are given in Table 1.

For the sake of clarity and simplicity of the general solutions in the different subdomains, we adopt the following notations:

$$
\begin{gathered}
P_{n}(x, y)=\left(\frac{x}{y}\right)^{n}+\left(\frac{x}{y}\right)^{-n} \\
E_{n}(x, y)=\left(\frac{x}{y}\right)^{n}-\left(\frac{y}{x}\right)^{n} \\
\Delta_{n}(x, y)=\left(\frac{x}{y}\right)^{2 n}-1
\end{gathered}
$$

A magnetic vector potential formulation is used in 2-D polar coordinates to describe the problem, the magnetic vector potential has only one component along the $\mathrm{z}$ direction and only depends on the $r$ and $\theta$ coordinates. The notations used in the paper are

$$
\begin{aligned}
& \boldsymbol{A}_{\boldsymbol{I}}=A_{I}(r, \theta) \cdot \boldsymbol{e}_{\boldsymbol{Z}} \text { for the inner PMs subdomain } \\
& \boldsymbol{A}_{I I}=A_{I I}(r, \theta) \cdot \boldsymbol{e}_{\boldsymbol{Z}} \text { for the inner air-gap subdomain } \\
& \boldsymbol{A}_{I I I}=A_{I I I}(r, \theta) \cdot \boldsymbol{e}_{\boldsymbol{Z}} \text { for the outer air-gap subdomain } \\
& \boldsymbol{A}_{I V}=A_{I V}(r, \theta) \cdot \boldsymbol{e}_{\boldsymbol{Z}} \text { for the outer PMs subdomain } \\
& \boldsymbol{A}_{\boldsymbol{i}}=A_{i}(r, \theta) \cdot \boldsymbol{e}_{\boldsymbol{Z}} \text { for the } i \text { th slot subdomain }
\end{aligned}
$$

\section{Analytical Solution}

\subsection{Mathematical models}

Based on the reasonable presumed conditions of the structure model of CMG, an ideal model can be proposed to analyze the magnetic gear. As shown in Fig. 1, the ideal model can be divided into five subdomains including the inner gap field, the outer gap field, the inner-rotor permanent magnet, the outer-rotor permanent magnet, and the modulating ring [14-15].

\subsection{The analysis of the magnetic field}

The solution of any partial differential equation (PDE) depends on the domain in which the solution is to be valid as well as the boundary conditions that the solution must satisfy. By using separation of variables, we now consider the solution of Laplace's equation for the slots and the air gap subdomains and the Poisson's equation for the PMs sub-domains.

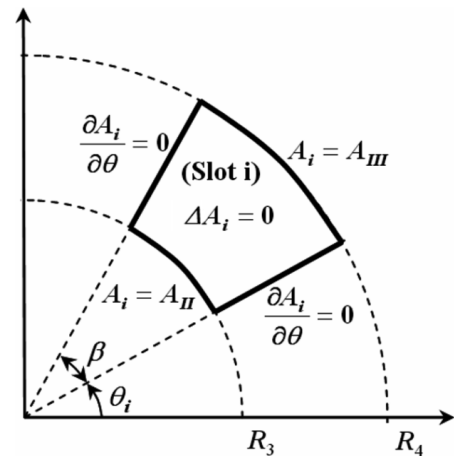

Fig. 2. $i$ th slot subdomain with its boundary conditions

\subsubsection{The ith slot subdomain}

The $i$ th slot subdomain and the associated boundary conditions are shown in Fig. 2. We have to solve the Laplace's equation in a domain of inner radius $R_{3}$ andouter radius $R_{4}$ delimited by the angles $\theta_{i}$ and $\theta_{i}+\beta$.

$$
\frac{\partial^{2} A_{i}}{\partial r^{2}}+\frac{1}{r} \frac{\partial A_{i}}{\partial r}+\frac{1}{r^{2}} \frac{\partial^{2} A_{i}}{\partial \theta^{2}}=0, R_{3} \leq r \leq R_{4}, \theta_{i} \leq \theta \leq \theta_{i}+\beta
$$

The boundary conditions for the $i$ th slot domain are

$$
\left\{\begin{array}{l}
A_{i}\left(R_{3}, \theta\right)=A_{\mathrm{II}}\left(R_{3}, \theta\right) \\
A_{i}\left(R_{4}, \theta\right)=A_{\mathrm{III}}\left(R_{4}, \theta\right) \\
\left.\frac{\partial A_{i}}{\partial \theta}\right|_{\theta=\theta_{i}}=0 \\
\left.\frac{\partial A_{i}}{\partial \theta}\right|_{\theta=\theta_{i}+\beta}=0
\end{array}\right.
$$

The general solution of (4) can be found by using the method of the separation of variables, the solution can be written as

$$
\begin{aligned}
A_{i}(r, \theta)= & A_{0}^{i}+B_{0}^{i} \ln r+\sum_{k=1}^{\infty}\left(\frac{R_{3}}{R_{4}}\right)^{\frac{k \pi}{\beta}}\left[A_{k}^{i} \frac{E_{k \pi / \beta}\left(r, R_{4}\right)}{\Delta_{k \pi / \beta}\left(R_{3}, R_{4}\right)}-\right. \\
& \left.B_{k}^{i} \frac{E_{k \pi / \beta}\left(r, R_{3}\right)}{\Delta_{k \pi / \beta}\left(R_{3}, R_{4}\right)}\right] \cos \left[\frac{k \pi}{\beta}\left(\theta-\theta_{i}\right)\right]
\end{aligned}
$$

where $k=1,2,3 \ldots, A_{0}^{i}$ is constants.

\subsubsection{Inner air-gap subdomain}

The inner air-gap subdomain and the associated boundary conditions are shown in Fig. 3. The problem to solve is

$$
\frac{\partial^{2} A_{I I}}{\partial r^{2}}+\frac{1}{r} \frac{\partial A_{I I}}{\partial r}+\frac{1}{r^{2}} \frac{\partial^{2} A_{I I}}{\partial \theta^{2}}=0, \quad R_{2} \leq r \leq R_{3}, 0 \leq \theta \leq 2 \pi
$$




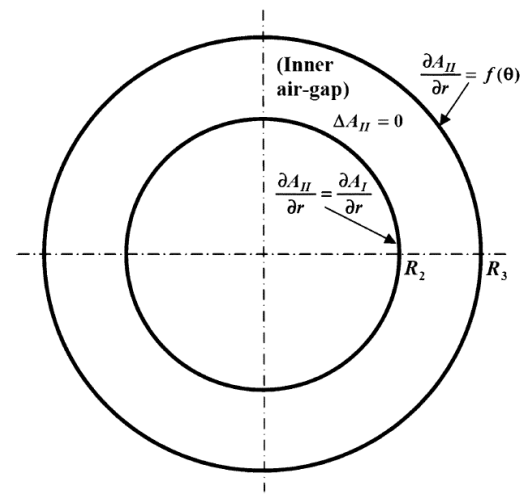

Fig. 3. Inner air gap subdomain (region II) with its boundary conditions

The boundary conditions for the inner air-gap are

$$
\left\{\begin{array}{l}
\left.\frac{\partial A_{\mathrm{II}}}{\partial r}\right|_{r=R_{2}}=\left.\frac{\partial A_{\mathrm{I}}}{\partial r}\right|_{r=R_{2}} \\
\left.\frac{\partial A_{\mathrm{II}}}{\partial r}\right|_{r=R_{3}}= \begin{cases}\left.\frac{\partial A_{i}}{\partial r}\right|_{r=R_{3}} & \theta_{i} \leq \theta \leq \theta_{i}+\beta \\
0 & \text { elsewhere }\end{cases}
\end{array}\right.
$$

The general solution of the magnetic vector potential in the inner air-gap can be written as

$$
\begin{gathered}
A_{\mathrm{II}}(r, \theta)=A_{0}^{\mathrm{II}}+\sum_{n=1}^{\infty}\left(\frac{R_{2}}{R_{3}}\right)^{n}\left[A_{n}^{\mathrm{II}} \frac{R_{2}}{n} \frac{P_{n}\left(r, R_{3}\right)}{\Delta_{n}\left(R_{2}, R_{3}\right)}-\right. \\
\left.B_{n}^{\mathrm{II}} \frac{R_{3}}{n} \frac{P_{n}\left(r, R_{2}\right)}{\Delta_{n}\left(R_{2}, R_{3}\right)}\right] \cos (n \theta)+\sum_{n=1}^{\infty}\left(\frac{R_{2}}{R_{3}}\right)^{n}\left[C_{n}^{\mathrm{II}} .\right. \\
\left.\frac{R_{2}}{n} \frac{P_{n}\left(r, R_{3}\right)}{\Delta_{n}\left(R_{2}, R_{3}\right)}-D_{n}^{\mathrm{II}} \frac{R_{3}}{n} \frac{P_{n}\left(r, R_{2}\right)}{\Delta_{n}\left(R_{2}, R_{3}\right)}\right] \sin (n \theta)
\end{gathered}
$$

Similarly, the expression of the magnetic vector potential of the outer gap field can be expressed as

$$
\begin{gathered}
A_{\mathrm{III}}(r, \theta)=A_{0}^{\mathrm{III}}+\sum_{n=1}^{\infty}\left(\frac{R_{4}}{R_{5}}\right)^{n}\left[A_{n}^{\mathrm{IIII}} \frac{R_{4}}{n} \frac{P_{n}\left(r, R_{5}\right)}{\Delta_{n}\left(R_{4}, R_{5}\right)}-\right. \\
\left.B_{n}^{\mathrm{III}} \frac{R_{5}}{n} \frac{P_{n}\left(r, R_{4}\right)}{\Delta_{n}\left(R_{4}, R_{5}\right)}\right] \cos (n \theta)+\sum_{n=1}^{\infty}\left(\frac{R_{4}}{R_{5}}\right)^{n} \\
{\left[C_{n}^{\mathrm{III}} \cdot \frac{R_{4}}{n} \frac{P_{n}\left(r, R_{5}\right)}{\Delta_{n}\left(R_{4}, R_{5}\right)}-D_{n}^{\mathrm{III}} \frac{R_{5}}{n} \frac{P_{n}\left(r, R_{4}\right)}{\Delta_{n}\left(R_{4}, R_{5}\right)}\right] \sin (n \theta)}
\end{gathered}
$$

\subsubsection{Inner rotor PMs subdomain}

The inner rotor PMs subdomain and the associated boundary conditions are shown in Fig. 4. The problem to solve is

$$
\begin{gathered}
\frac{\partial^{2} A_{\mathrm{I}}}{\partial r^{2}}+\frac{1}{r} \frac{\partial A_{\mathrm{I}}}{\partial r}+\frac{1}{r^{2}} \frac{\partial^{2} A_{\mathrm{I}}}{\partial \theta^{2}}=\frac{\mu_{0}}{r} \frac{\partial M_{\mathrm{r}}}{\partial \theta}, \\
R_{1} \leq r \leq R_{2}, 0 \leq \theta \leq 2 \pi
\end{gathered}
$$

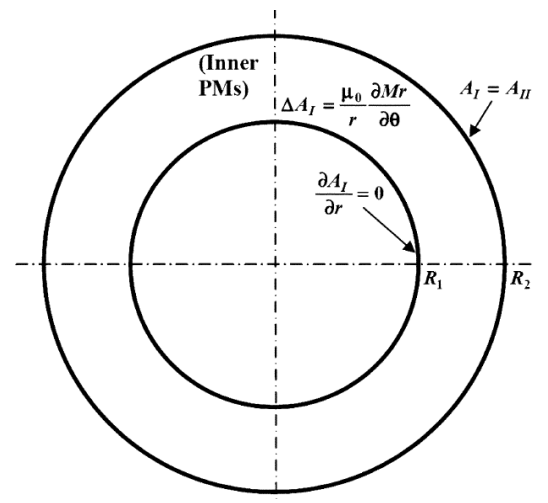

Fig. 4. Inner PMs subdomain (region I) with its boundary conditions

where $\mu_{0}$ is the permeability of the vacuum and $\mathrm{M}_{\mathrm{r}}$ is the radial magnetization of the magnets.

The boundary conditions for the inner rotor PMs subdomain

$$
\left\{\begin{array}{l}
\left.\frac{\partial A_{\mathrm{I}}}{\partial r}\right|_{r=R_{1}}=0 \\
\left.\frac{\partial A_{\mathrm{I}}}{\partial \theta}\right|_{r=R_{2}}=\left.\frac{\partial A_{\mathrm{II}}}{\partial \theta}\right|_{r=R_{2}}
\end{array}\right.
$$

The general solution of the magnetic vector potential in the inner PMs subdomain can be written as

$$
\begin{gathered}
A_{\mathrm{I}}(r, \theta)=\sum_{n=1}^{\infty}\left[\left(\frac{R_{1}}{R_{2}}\right)^{n} A_{n}^{\mathrm{I}} \frac{P_{n}\left(r, R_{1}\right)}{\left(R_{1} / R_{2}\right)^{2 n}+1}+\right. \\
\left.U_{n}(r) \cos \left(n \phi_{\mathrm{i}}\right)\right] \cos (n \theta)+\sum_{n=1}^{\infty}\left[\left(\frac{R_{1}}{R_{2}}\right)^{n} C_{n}^{\mathrm{I}} .\right. \\
\left.\frac{P_{n}\left(r, R_{1}\right)}{\left(R_{1} / R_{2}\right)^{2 n}+1}+U_{n}(r) \sin \left(n \phi_{\mathrm{i}}\right)\right] \sin (n \theta)
\end{gathered}
$$

where

$$
U_{n}(r)=\left\{\begin{array}{l}
0, \text { others } \\
\frac{4 B_{\mathrm{r}} p_{\mathrm{i}}}{\pi\left(1-n^{2}\right)}\left\{1+\frac{R_{1}}{n}\left(\frac{R_{1}}{r}\right)^{n}-\frac{P_{n}\left(r, R_{1}\right)}{\left(\frac{R_{1}}{R_{2}}\right)^{2 n}+1}\right. \\
\left.\left[\left(\frac{R_{1}}{R_{2}}\right)^{n}+\frac{R_{1}}{n}\left(\frac{R_{1}}{R_{2}}\right)^{2 n}\right]\right\}, n=j p_{\mathrm{i}}, j=1,3,5 \ldots
\end{array}\right.
$$

where $n$ is a positive integer, $P_{i}$ is the number of pole-pairs of the inner rotor.

Similarly, the expression of the magnetic vector potential of the outer PMs subdomain can be expressed as 


$$
\begin{gathered}
A_{\mathrm{IV}}(r, \theta)=\sum_{n=1}^{\infty}\left[\left(\frac{R_{5}}{R_{6}}\right)^{n} A_{n}^{\mathrm{IV}} \frac{P_{n}\left(r, R_{6}\right)}{\left(R_{5} / R_{6}\right)^{2 n}+1}+V_{n}(r) .\right. \\
\left.\cos \left(n \phi_{\mathrm{o}}\right)\right] \cos (n \theta)+\sum_{n=1}^{\infty}\left[\left(\frac{R_{5}}{R_{6}}\right)^{n} C_{n}^{\mathrm{IV}} .\right. \\
\left.\frac{P_{n}\left(r, R_{6}\right)}{\left(R_{5} / R_{6}\right)^{2 n}+1}+V_{n}(r) \sin \left(n \phi_{\mathrm{o}}\right)\right] \sin (n \theta)
\end{gathered}
$$

where

$$
V_{n}(r)=\left\{\begin{array}{l}
0, \text { others } \\
\frac{4 B_{\mathrm{r}} p_{\mathrm{o}}}{\pi\left(1-n^{2}\right)}\left\{1+\frac{R_{6}}{n}\left(\frac{R_{6}}{r}\right)^{n}-\frac{P_{n}\left(r, R_{6}\right)}{\left(\frac{R_{5}}{R_{6}}\right)^{2 n}+1}\left[\left(\frac{R_{5}}{R_{6}}\right)^{n}+\right.\right. \\
\left.\left.\frac{R_{6}}{n}\left(\frac{R_{6}}{R_{5}}\right)^{2 n}\right]\right\}, n=j p_{\mathrm{o}}, j=1,3,5 \ldots
\end{array}\right.
$$

where $P_{o}$ is the number of pole-pairs of the outer rotor. $A_{0}^{I I}, A_{n}^{I I}, B_{n}^{I I}, C_{n}^{I I}, D_{n}^{I I}, A_{0}^{I I I}, A_{n}^{I I I}, B_{n}^{I I I}, C_{n}^{I I I}, D_{n}^{I I I}, A_{0}^{i}, B_{0}^{i}, A_{k}^{i}, B_{k}^{i}$ are constants, Taking the partial derivatives of expression (9) and (10) with respect to the radius respectively, the radial flux density $B_{r}$ can be obtained. Similarly, the tangential flux density $B_{\theta}$ can be also obtained.

\subsection{Electromagnetic torque}

The electromagnetic torque is obtained using the Maxwell Stress tensor. A circle of radius $r$ in the air gap is taken as the integration path so the electromagnetic torque is expressed as follows:

$$
T_{\mathrm{em}}=\frac{L_{\mathrm{ef}}}{\mu_{0}} \int_{0}^{2 \pi} r^{2} B_{r} B_{\theta} \mathrm{d} \theta
$$

where, $L_{e f}$ refers to the axial length, $r$ refers to the radius of any circumference in the air gap, $B_{r}, B_{\theta}$ refers to the radial component and the tangential component of the gas flux density respectively. For the selected radius, $r$ is the constant and $\mu_{0}$ is the permeability of vacuum.

\section{The Fundamental Principles and Steps of Genetic Algorithm}

Genetic Algorithm is a branch of the simulated optimization method which belongs to the random global optimization method. The fundamental principle of Genetic Algorithm bases on the natural genetic mechanism and the natural selection mechanism in nature, simulates the rules of the survival of the fittest and the genetic operation, optimizes the heuristic evolutionary operation by using the fitness function of individual in a group. The main calculation process of Genetic Algorithm is expressed as following:
1) Generate an initial group randomly, which is constituted by the initial individuals. Each individual refers to a genetic code of chromosome.

2) Calculate the fitness of each individual chromosome in the group, and judge whether it meets the need of the optimization criterion.

3) According to the fitness, choose the regenerative individual. If the fitness of an individual is high, it is easy to be chosen as the regenerative individual, and vise verse.

4) According to certain crossover probability and crossover method, generate a new individual.

5) According to certain mutation probability and mutation method, generate a new individual.

6) The new group generated through the genetic operatorscrossover and mutation go back to step (2).

There are many methods adopted to design the traditional electric motors, such as the complex method, the penalty function method, the Mountain climbing algorithm and so on. These methods are applied success-fully to varying degrees, but they have their own disadvantages at the same time, such as being apt to get to its local optimum point, the optimization results are related to the choice of the initial point, being difficult to handle the discrete variables. Comparing with the traditional methods, Genetic Algorithm is a global optimization method with many advantages. It not only has strong robustness, but also has the ability to handle continuous and discrete variables effectively. It does not request the objective function and its constraints to be differentiable and continuous and at the same time, its region of search can go through the whole solution space. Therefore, this paper adopts the genetic algorithm optimization toolbox of matlab (GAOT) to optimize the parameters of the CMG [16].

The main function of GAOT is expressed as:

[x fval]=ga(@fitnessfcn, nvars, options)

The output parameters:

$\mathrm{x}$ : Returns the end point.

fval: The value of the fitness function at $\mathrm{x}$ point.

The input parameters:

(a)fitnessfen: The function handles of File M to calculate the fitness function.

nvars: The numbers of variables in the fitness function. options: The structural body of the parameters.

As the optimization design of $\mathrm{CMG}$ has constraint problems, it is not right to pursue the optimization of the objective function. The optimization result is restricted by the conditions as the structure and technical characteristics.

\section{The Optimization Example}

\subsection{The objective function}

This paper adopts the ratio of the electromagnetic torque 
to the volume as the objective function to solve the optimization problem of the concentric magnetic gear:

$$
\max f(\boldsymbol{X})=\frac{T_{e m}}{V}
$$

where $V$ is the volume of the magnetic gear, which we expect to remain unchanged. The objective function transforms into a function which makes the electromagnetic torque to maximize.

\subsection{The optimization variables}

Choose six parameters which have greater influence on the torque of the concentric magnetic gear as the optimization variables:

$$
\boldsymbol{X}=\left[\beta, R_{1}, R_{2}, R_{3}, R_{4}, R_{5}\right]^{T}
$$

where $\beta$ is the width of the slot.

\subsection{The constraints}

The main constraint: The bigger the largest average flux density of the adjustable magnetic ring is, the more the iron loss is. As the iron loss does not take a dominate place, then the value range of Bps can be expressed as $B_{p s} \leq 1.8 \mathrm{~T}$.

Set the largest average flux density of the rotors as $B_{c s} \leq 1.5 \mathrm{~T}$ to avoid the magnetic saturation by taking consideration of the structural strength in the yoke of the two rotor cores.

In addition, there are constraints about the structure of the magnetic gear and its craftsmanship.

\subsection{The optimization example}

This paper optimizes the $\mathrm{CMG}$ with the following parameters: $P_{\text {in }}=4$ (pole-pairs inner rotor), $P_{\text {out }}=17$ (polepairs outer rotor), $\mathrm{Q}=21$ (number of ferromagnetic polepieces), $B_{r}=1.1 \mathrm{~T}, L_{e f}=40 \mathrm{~mm}$, the harmonic order of the gas magnetic field is 150 ; the harmonic order of the slot field is 30 . The group size is 30 , the iteration is 80 , the roulette method is adopted to choose functions, the cross function wants a linear recombination with the probability being 0.9 . The variant function requires uniform mutation with the probability being 0.04 . The optimization results are shown in Table 2.

Table 2. Example of GA optimization

\begin{tabular}{c|c|c}
\hline Optimization variables & initial value & optimization \\
\hline$R_{1}(\mathrm{~mm})$ & 60 & 64.9 \\
\hline$R_{2}(\mathrm{~mm})$ & 70 & 75.1 \\
\hline$R_{3}(\mathrm{~mm})$ & 71 & 75.9 \\
\hline$R_{4}(\mathrm{~mm})$ & 86 & 85.3 \\
\hline$R_{5}(\mathrm{~mm})$ & 87 & 86.1 \\
\hline$\beta(\mathrm{rad})$ & 0.1496 & 0.1408 \\
\hline
\end{tabular}

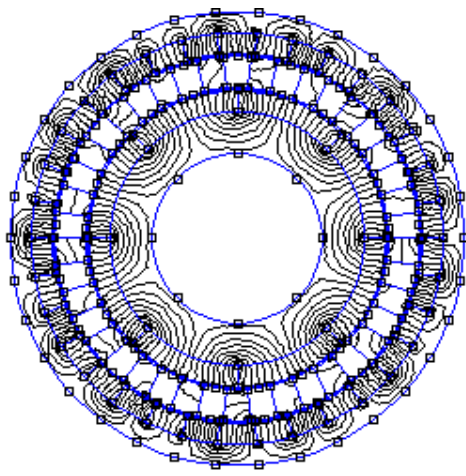

Fig. 5. Magnetic field distributions

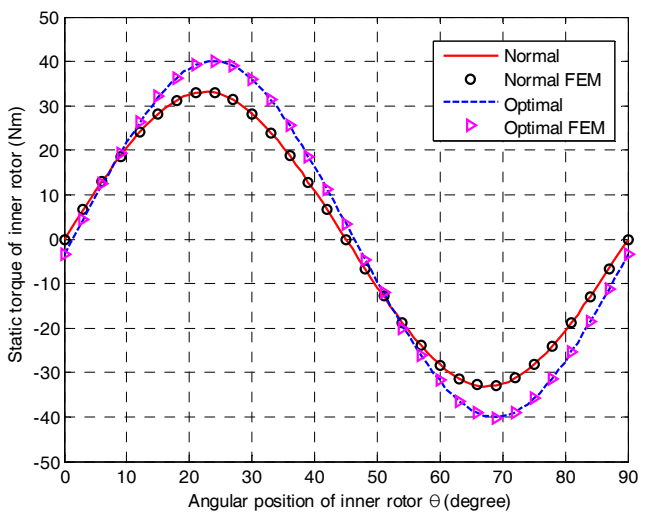

(a)

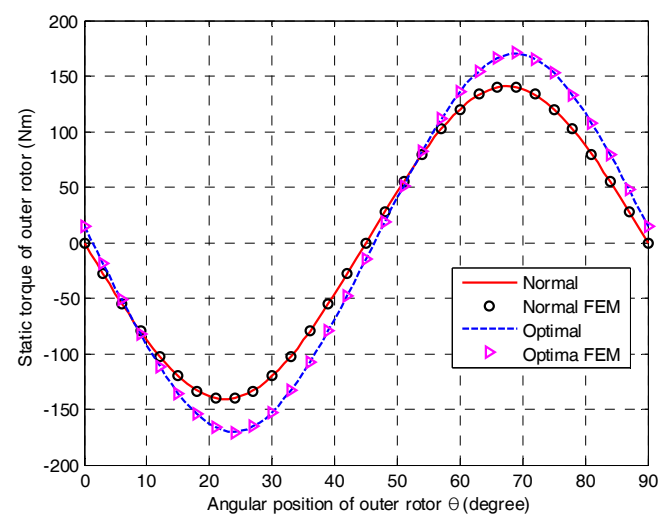

(b)

Fig. 6. Torque-angle curve (a) Inner rotor (b) Outer rotor

Fig. 5 shows the magnetic flux lines for the magnetic gear excited by both permanent magnet rotors.

Fig. 6 shows the waveform about the static electromagnetic torque of the inner and outer rotors before and after the optimization of the CMG.

As shown in Fig.6, the curve of the magnetic torque after the optimization is closer to sine wave and the ratio of $-1: 4.25$, which accords with the drive ratio of the concentric magnetic gear, remains in the same proportion between the inner magnetic torque and the outer magnetic torque The maximum magnetic torque of the inner rotor is $40.1 \mathrm{~N} \cdot \mathrm{m}$ after the optimization while it is $33.1 \mathrm{~N} \cdot \mathrm{m}$ before 


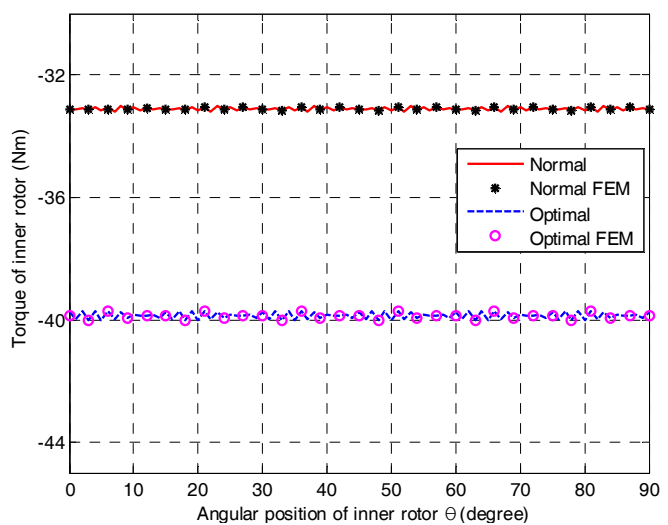

(a)

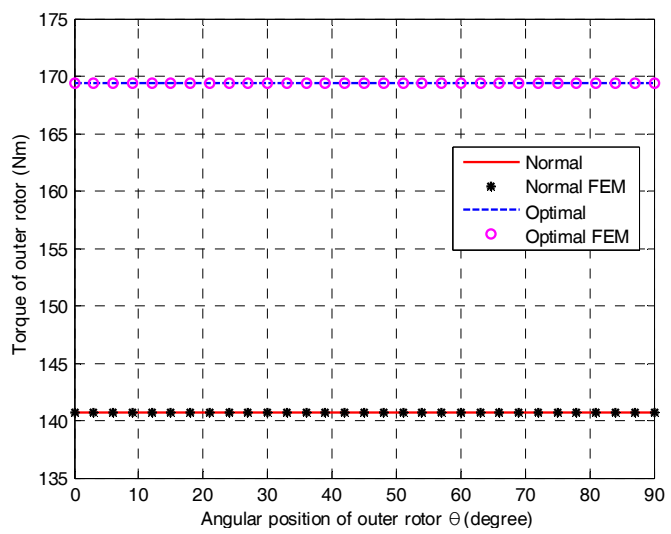

(b)

Fig. 7. Electromagnetic torque exerted: (a) on the inner rotor; (b) on the outer rotor

the optimization. During this process, the torque is increased by $21 \%$. The maximum magnetic torque of the outer rotor is $170.1 \mathrm{~N} \cdot \mathrm{m}$ after the optimization while it is $140.6 \mathrm{~N} \cdot \mathrm{m}$ before the optimization. During this process, the torque is increased by $21 \%$, too. Therefore, the torque density of the concentric magnetic torque is increased by $13 \%$ after the optimization.

Fig. 7 compares the output torques between the inner rotor and the outer rotor after the optimization. At this time, the inner rotor rotates clockwise while the outer rotor rotates anticlockwise after fixing the adjustable magnetic ring, and satisfies the relation:

$$
\varphi_{\mathrm{o}}=-\varphi_{i} \cdot \frac{p_{i}}{p_{o}}
$$

The electromagnetic torque ripple of the concentric magnetic gear is generated by the flux density harmonics, especially the low-order harmonics of the two layers of the air gas field. In order to reduce the torque ripple, it is necessary to choose a proper drive ratio of the magnetic gear that is to choose proper $P_{\mathrm{o}}$ and Q [17].

The manufactured CMG is shown in Fig. 8 and the test bench is shown in Fig. 9.
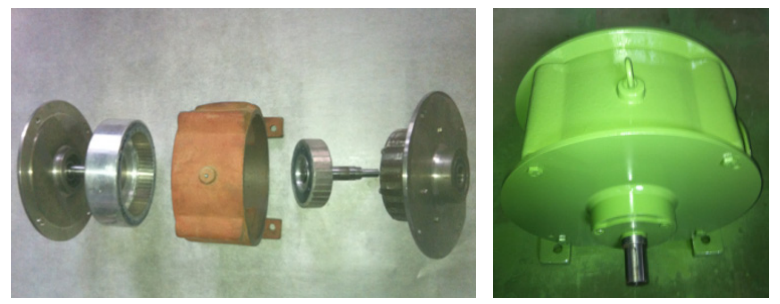

Fig. 8. The manufactured $\mathrm{CMG}$

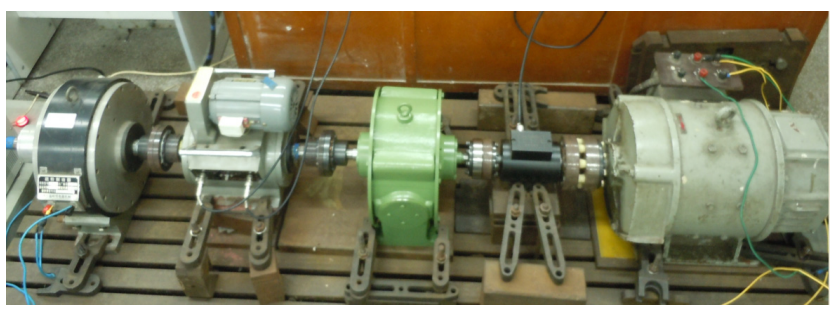

Fig. 9. Test bench

The efficiency of the CMG at a constant speed of $150 \mathrm{r} / \mathrm{min}$ on outer rotor, and the experimental data of load are given in Table 3.

Table 3. The efficiency of load side for $100 \mathrm{r} / \mathrm{min}$

\begin{tabular}{c|c|c}
\hline Input torque $/(\mathrm{N} \cdot \mathrm{m})$ & Output torque/(N·m) & Efficiency/(\%) \\
\hline 2.1 & 8.2 & $66.5 \%$ \\
\hline 5.05 & 19.7 & $83 \%$ \\
\hline 10 & 40 & $87.5 \%$ \\
\hline 12.4 & 50.86 & $89 \%$ \\
\hline 14.69 & 60.4 & $90.4 \%$ \\
\hline 17.17 & 70.6 & $91.6 \%$ \\
\hline 18 & 74 & $92.1 \%$ \\
\hline
\end{tabular}

From the prototype CMG with load test results, the efficiency of CMG is decided by the size of load, because of the CMG's load loss and no-load loss is basically consistent, so the light load leads to CMG's efficiency is low. With the rising of rotating speed, the loss will increase, so the transmission efficiency will decrease. When load torque is greater than $60 \mathrm{~N} \cdot \mathrm{m}$, its efficiency is above $90 \%$. When the load torque is $74 \mathrm{~N} \cdot \mathrm{m}$, the corresponding output torque density is $57.18 \mathrm{kN} \cdot \mathrm{m} / \mathrm{m}^{3}$.

\section{Conclusion}

This paper analyzes and calculates the electromagnetic field and the electromagnetic torque of the concentric magnetic gear by adopting the exact analytical method, and optimizes the design of a concentric magnetic gear based on Genetic Algorithm Toolbox of Matlab. The optimization results indicate that the magnetic torque becomes bigger comparing with the initial one, the drive ratio of the magnetic gear remains as before, the torque density increases significantly. It offers an effective method for the transmission gear with low rotate speed and high torque. 


\section{Acknowledgements}

This work was supported by Hubei Key Laboratory of Hydroelectric Machinery Design \& Maintenance (2016 KJX14), the talent foundation of China Three Gorges University (KJ2013B078), and the Graduate Innovation Foundation of China Three Gorges University (2015CX060, SDYC2016047). At the same time, we thank Shanghai University for assistance.

\section{References}

[1] T. Haring, K. Forsman, T. Huhtanen, and M. Zawadzki, "Direct Drive-Opening a New Era in Many Applications," Pulp and Paper Industry Technical Conference, pp. 171-179, 2003.

[2] D. H. Kang, Y. H. Chun, and H. Weh, "Analysis and optimal design of transverse flux linear motor with PM excitation for railway traction," IEE Proceedings - Electric Power Applications , Vol. 150, No. 4, pp. 493-499, 2003.

[3] M. G. Simoes and P. J. Vieira, "A high-torque lowspeed multiphase brushless machine-a perspective application for electric vehicles," IEEE Trans. Ind. Appl., Vol. 49, No. 5, pp.1154-1164,2002.

[4] G. H. Feng, L. F. Wang and B. Y, "Zhang. Analysis of magnetic field for low speed and high torque permanent magnet synchronous machine," Sixth International Conference on Electrical Machines and Systems, ICEMS, PP. 778-781, 2003.

[5] L. N. Jian, K. T. Chau and J. Z. Jiang, "A magneticgeared outer-rotor permanent-magnet brushless machine for wind power generation," IEEE Trans. Ind. Appl., Vol. 45, No. 3, pp.954-962, 2009.

[6] B. C. Polinder and A. G. Mecrow, "Conventional and TFPM Linear Generators for Direct-Drive Wave Energy Conversion," IEEE Trans. Energy Conversion, Vol. 20, No. 2, pp. 260-267, 2005.

[7] Z. Q. Zhu and D. Howe, "Instantaneous magneticfield distribution in brushless permanent-magnet $\mathrm{dc}$ motor, part III: Effect of slotting," IEEE Trans. Magn., Vol. 29, No. 1, pp. 143-151, 1993.

[8] D. Zarko, D. Ban and T. A. Lipo, "Analytical calculation of magnetic field distribution in the slotted air gap of a surface permanent-magnet motor using complex relative air-gap permeance," IEEE Trans. Magn., Vol. 42, No. 7, pp.1828-1837, 2006.

[9] Z. J. Liu and J. T. Li, "Analytical solution of air-gap field in permanent magnet motors taking into account the effect of pole transition over slots," IEEE Trans. Magn., Vol. 43, No. 10, pp.3872-3882, 2007.

[10] T. Lubin, S. Mezani and A. Rezzoug, "Exact analytical method for magnetic field computation in the air-gap of cylindrical electrical machines considering slotting effects," IEEE Trans. Magn., Vol. 46, No. 4, pp. 1092-1099, 2010.

[11] P. R. Upadhyay and K. R. Rajagopal, "Genetic algorithm based design optimization of a permanent magnet brushless dc motor," Journal of Applied Physics, Vol. 97, No. 10, pp. 10Q516-10Q516-3, 2005.

[12] S. L. Ho, N. N. Chen and W. N. Fu, "An optimal design method for the minimization of cogging torques of a permanent magnet motor using FEM and Genetic Algorithm," IEEE Trans. Appl. Supercond., Vol. 40, No. 3, pp.861-864, 2010.

[13] A. Mahmoudi, S. Kahourzade and N. A. Rahim, "Design, analysis, and prototyping of an axial-flux permanent magnet motor based on genetic algorithm and finite-element analysis," IEEE Trans. Magn., Vol. 49, No. 4, pp.1479-1492, 2013.

[14] T. Lubin, S. Mezani and A. Rezzoug, "Analytical computation of the magnetic field distribution in a magnetic gear," IEEE Trans. Magn., Vol. 46, No. 7, pp. 2611-2621, 2010.

[15] S. Q. Du, J. Z. Jiang and Y. J. Zhang, "A magnetic gearing," Diangong Jishu Xuebao / Transactions of China Electro technical Society, Vol. 25, No. 9, pp. 41-46, 2010.

[16] D. R. Wu, J. C. Li and Li, Q. F, "Design optimization of series electrical machines using genetic algorithms," Journal of Xi'an Jiaotong University, Vol. 23, No. 2, pp. 14-18, 1999.

[17] K. Atallah and D. Howe, "A novel high-performance magnetic gear," IEEE Trans. Magn., 2001, Vol. 37, No. 4, pp. 2844-2846, 2001.

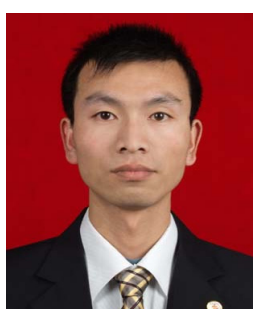

Libing Jing received his B.Sdegree from Zhongyuan University of Technology in 2006 and the Ph.D. degree from Shanghai University in 2013. He is currently an associate professor in the College of Electrical Engineering and New Energy, China Three Gorges University, His research activities are related to design, modeling, and analysis of electrical machines.

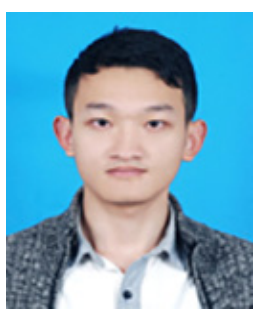

Zhenghao Luo received B.S degree in Nanchang Institute of Technology in 2013. He is currently working towards the M.S. in China Three Gorges University. His research interests include optimization of electrical machines finite element analysis. 


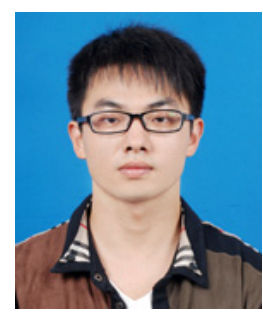

Lin Liu received B.S degree in electrical engineering from China Three Gorges University in 2014. He is currently working towards the M.S. in China Three Gorges University. His research interests include optimization of electrical machines finite element analysis.

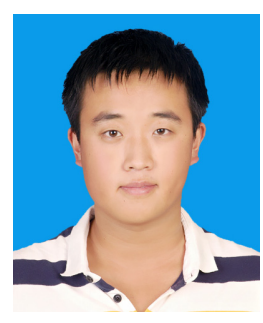

Qixing Gao received his B.S. degree in Department of Electrical Engineering in Hebei University of Architecture in 2015. He is currently working towards the M.S. in China Three Gorges University in China. His research interests include optimization of electrical machines finite element analysis. 